\title{
Effects of short-term isokinetic training with reciprocal knee extensors agonist and antagonist muscle actions: A controlled and randomized trial
}

\author{
Rafael Cunha ${ }^{1}$, Rodrigo L. Carregaro ${ }^{1,2}$, André Martorelli ${ }^{1}$, \\ Amilton Vieira $^{1}$, Ana B. Oliveira ${ }^{3}$, Martim Bottaro ${ }^{1}$
}

\begin{abstract}
Background: Previous studies have shown that preloading an antagonist muscle may increase the acute agonist neuromuscular performance. In addition, studies have suggested that very short-term resistance exercise (RE) programs may also be useful to increase strength and muscular performance. Objectives: To evaluate the effects of three days of RE using a reciprocal action method on the muscular performance of healthy men and to compare these effects with those of a traditional RE group. Method: Thirty-three men (21.1 \pm 2.3 years) were randomly assigned to one of three groups: 1) reciprocal (REC; knee flexion immediately followed by a knee extension exercise); 2) traditional (TRA; nonpreload; a concentric knee extension exercise); and 3) control (CON; no exercise). The REC and TRA subjects performed four sets of 10 repetitions at $60 \%$ s with one minute of rest. The pre- and post-RE tests included two sets of four maximal concentric repetitions at $60 \%$ s and $180 \%$ s. A 3x2 ANOVA with Tukey post-hoc was used to analyze the differences in peak torque (PT), rating of acceleration development (RAD) and time to peak torque (TIME $\left.{ }_{\text {torque }}\right)$. Results: A significant PT increase was found for REC and TRA $(p<0.05)$ at $60 \%$ and for REC at $180 \% \mathrm{~s}(p<0.05)$. There was a decrease in the RAD for REC and TRA $(p<0.05)$, and TIME ${ }_{\text {torque }}$ showed a significant decrease for REC. The inter-group analysis revealed that REC is more effective than TRA for PT gains at both velocities $(p<0.05)$. Conclusion: It is recommended that REC offers benefits for the clinical practice of professionals involved in neuromuscular rehabilitation.
\end{abstract}

Keywords: muscle strength; rehabilitation; knee; resistance training.

\section{HOW TO CITE THIS ARTICLE}

Cunha R, Carregaro RL, Martorelli A, Vieira A, Oliveira AB, Bottaro M. Effects of short-term isokinetic training of the reciprocal knee extensors agonist and antagonist muscle actions: A controlled and randomized study. Braz J Phys Ther. 2013 Mar-Apr; 17(2):137-145. http://dx.doi.org/10.1590/S1413-35552012005000077

\section{Introduction}

Muscular strength gains from resistance exercise (RE) programs are responsible for improving velocity and movement coordination ${ }^{1,2}$, in addition to promoting balance and functionality ${ }^{3}$. These findings highlight the importance of implementing RE in the context of human health, and it is currently recommended as part of physical activity programs for adults ${ }^{4}$, seniors ${ }^{4}$ and heart disease patients ${ }^{4,5}$. Traditionally, an eight-week program is necessary to achieve significant and visible improvements in strength and muscular performance ${ }^{6}$, although gains can be observed prior to this period ${ }^{7}$. Several studies have focused on the strength gains from short duration
RE sessions, which vary from two to three sessions per week for up to two weeks ${ }^{8-11}$. Prevost et al. ${ }^{8}$ found an increase in peak torque (PT) after shortterm training that was similar to the gains achieved after 6 to 10 weeks of training. This finding suggests the possibility of combining the effectiveness of the results provided by $\mathrm{RE}$ with a smaller number of sessions, which might improve athletic performance and achieve rehabilitation of the musculoskeletal function. This protocol may make a difference in the willingness of individuals who are performing a rehabilitation process or training, especially if their initial goals are met in a limited number of visits ${ }^{11}$.

\footnotetext{
${ }^{1}$ Strength Training Laboratory, School of Physical Education, Universidade de Brasília (UnB), Brasília, DF, Brazil 
RE performed in the isokinetic dynamometer shows important advantages that can be useful in various parts of the evaluation process and during rehabilitation. The force exerted by the subject is proportionally returned in the form of resistance in the isokinetic dynamometer. Therefore, a maximum level of muscular performance is required across the entire range of motion (ROM). In contrast, the maximum tension occurs only at the most favorable mechanical point of the $\mathrm{ROM}^{12}$ in isoinertial actions. The analysis of muscle function under several conditions and with highly reliable measurements makes the isokinetic dynamometer a valid and widely used tool in both physical therapy and sports ${ }^{16}$.

The use of reciprocal muscle contractions between antagonists and agonists is one of the RE methods that has attracted increasing interest for rehabilitation, and it consists of performing an antagonistic concentric muscle action immediately before an agonistic concentric action for each repetition of the exercise ${ }^{17-22}$. Cross-sectional studies have demonstrated a greater capacity for work using reciprocal action methods ${ }^{18,20,23,24}$.

Strength gain and improvements in neuromuscular performance during the early stages of RE, coupled with findings from reciprocal action methods, may help health professionals involved in rehabilitation and physical performance to design exercise prescriptions ${ }^{11,16,25}$. Therefore, the objective of this study was to evaluate the effects of a short-term RE program (three sessions) using reciprocal contraction on the neuromuscular responses of healthy young men compared with a short traditional RE program. The hypothesis is that a short-term RE program accomplished through reciprocal actions is more efficient for strength gain and muscle performance than the traditional short-term training carried out only by agonist actions.

\section{Method}

\section{Sample}

Thirty-three male college students who were physically active but who had not practiced RE for at least six months were recruited. The demographics of the participants are shown in Table 1. Volunteers were recruited through flyers placed around the campus, and verbal invitations were provided in classrooms. The following criteria was used for inclusion in the study: no cardiorespiratory compromise; no musculoskeletal and joint injury; no metabolic disease and no participation in any RE program at least six months preceding the start of the experiment. The participants who met the criteria answered a questionnaire on their health. They were then invited to participate and signed a consent form that informed them of the objectives and procedures used in the study, according to resolution 196/96 of the National Health Council (Conselho Nacional de Saúde (CNS)), approved by the Ethics Committee of the School of Health at Universidade de Brasília (UNB), Brasília, DF, Brazil, under protocol number 161/2008.

All of the procedures were performed at the institutional laboratory between February and May of 2011. The subjects who were selected to participate in the present study were admitted sequentially and then randomly divided in three groups: a control group (CON), a reciprocal exercise group (REC) and a traditional group (TRA). A sealed opaque envelope containing several cards with the names of the various interventions was used. The names CONTROL, RECIPROCAL and TRADITIONAL were used, ensuring the confidentiality of the allocation for each participant. A researcher who was unaware of the goals or purpose of the study performed this procedure. Preliminary statistical analysis revealed

Table 1. Demographic characteristics of the participants. Data are shown as the mean \pm standard deviation.

\begin{tabular}{lcccc}
\hline \multicolumn{1}{c}{ Variable } & Experimental Group & & $p$ \\
Age $(\mathrm{y})$ & $\mathbf{C O N}(\mathbf{n}=\mathbf{8})$ & TRA $(\mathbf{n = 1 1})$ & RA $(\mathbf{n = 1 0})$ & 0.48 \\
Height $(\mathrm{m})$ & $20.88 \pm 1.96$ & $20.50 \pm 2.76$ & $21.73 \pm 2.15$ & 0.46 \\
Body mass $(\mathrm{kg})$ & $1.75 \pm 0.06$ & $1.78 \pm 0.06$ & $1.76 \pm 0.05$ & 0.40 \\
BMI $\left(\mathrm{kg} / \mathrm{m}^{2}\right)$ & $75.46 \pm 7.99$ & $70.15 \pm 9.85$ & $74.70 \pm 9.33$ & 0.07 \\
\hline
\end{tabular}

CON: control, TRA: traditional, REC: reciprocal, BMI: body mass index. 


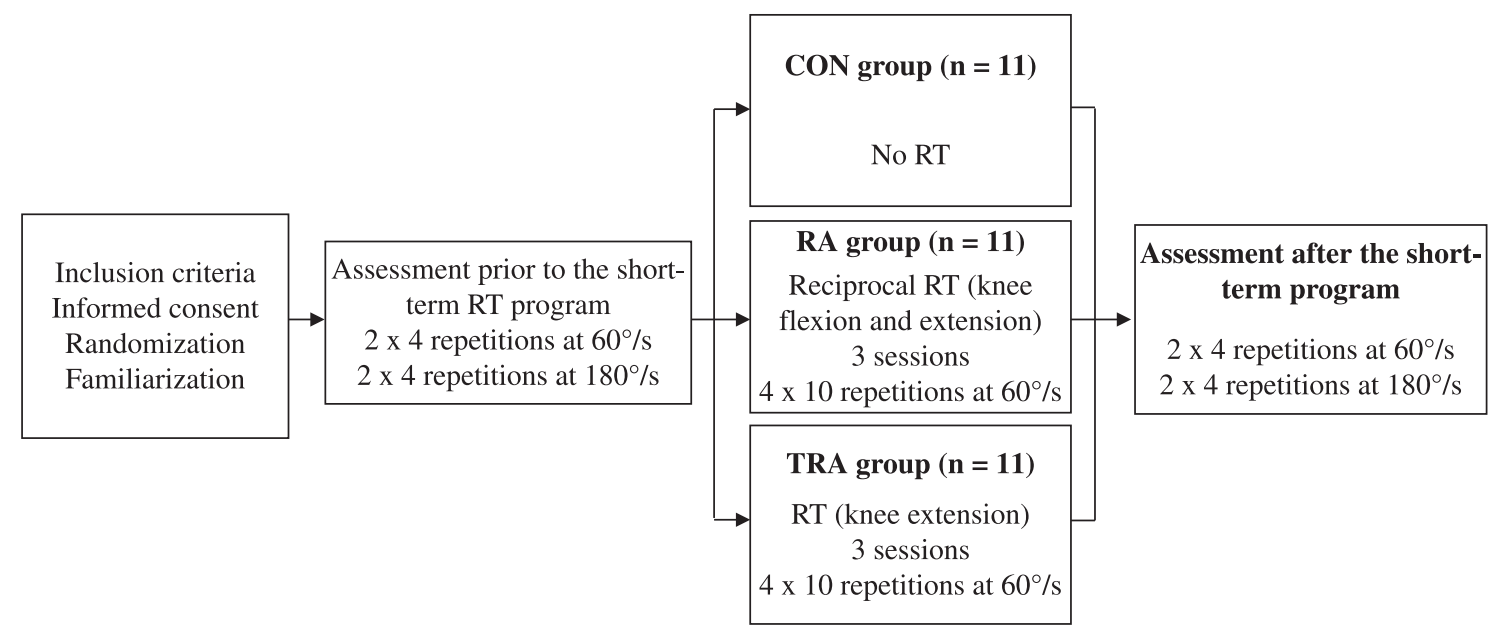

Figure 1. Experimental design.

that the anthropometric profile was similar among the participants of each group ( $p>0.05$ ). A schematic of the study is shown in Figure 1.

\section{Isokinetic dynamometry}

The torque and neuromuscular performance of the knee extensors were obtained using an isokinetic dynamometer, model Biodex System III (Biodex Medical, Inc., Shirley, NY). The calibration and positioning procedures followed the manufacturer's specifications. The right lower limb was evaluated, as previous studies ${ }^{26}$ found no difference in the isokinetic variables between the dominant and nondominant limb of untrained individuals. The subjects sat in the equipment chair in a manner that allowed the rotational axis of the dynamometer to remain aligned with the lateral epicondyle of the femur during the tests. The lever arm was adjusted and fixed $5 \mathrm{~cm}$ above the calcaneus. The chair and dynamometer adjustments for each subject were recorded to ensure that the position remained constant throughout the testing period. The subjects were stabilized in the equipment using restraints on their thighs, pelvis and trunk to prevent unwanted movement. A gravity correction was obtained by measuring the torque exerted by the resistance arm and leg of the subject (relaxed) in the terminal extension position. During the test, the volunteers were asked to keep their arms crossed over their thoracic region ${ }^{27}$. Verbal encouragement and visual feedback through the dynamometer computer monitor were provided to encourage the subjects to reach their maximum exertion level. The same investigator performed all of the tests for all of the subjects.

\section{Evaluation procedures}

Prior to the data collection, the subjects were instructed to avoid consuming food or stimulant drinks and to avoid performing any strenuous exercise throughout the experiment duration. After reading and understanding all of the research procedures, the volunteers watched a video describing the test procedures and exercises. The subjects then underwent a familiarization process (Figure 1).

The tests to evaluate PT before and after the RE program consisted of two sets of four maximum repetitions of isokinetic concentric contractions of the knee at $60 \%$ s and $180 \%$ s, with a one-minute rest interval between the sets and a two-minute rest interval between each velocity ${ }^{28}$. The rate of acceleration development (RAD) and the time to reach PT (TEMP $\left.{ }_{\text {torque }}\right)$ was only recorded at the lowest velocity $\left(60^{\circ} \%\right)$ ). A ROM of $80^{\circ}$ flexo-extension (excursion between $10^{\circ}$ and $90^{\circ}$ relative to a $0^{\circ}$ full knee extension) was used. All of the evaluations were preceded by a series of warm ups, which consisted of four submaximal repetitions at $120 \%$ s.

\section{Isokinetic training}

The subjects in the reciprocal group (REC, $n=11)$ underwent a three-session isokinetic concentric reciprocal knee flexors and extensors training protocol. The subjects in the traditional group (TRA, $\mathrm{n}=11$ ) underwent a three-session isokinetic concentric 
knee extensors protocol. The subjects in the control group (CON, $\mathrm{n}=11)$ were only subjected to pre- and post-training evaluations.

Each training session was separated by an interval of 72 hours and consisted of four series of ten concentric isokinetic repetitions at $60 \%$ with a one-minute rest interval between sets. The number of series and sets followed the recommendations of the American College of Sports Medicine for muscle strength gain ${ }^{4}$, and velocity was selected because it is often used to assess the isokinetic actions of healthy young people ${ }^{29}$.

\section{Data processing}

Data processing was performed using MatLab ${ }^{\circledR}$ (version 7.8 release 2009a, MathWorks Inc., USA). In the present study, only the repetition with the highest PT value was evaluated. The RAD values were calculated based on the slope of the velocity versus time curve during the initial phase of concentric isokinetic contractions. RAD, therefore, represents the time taken to achieve the isokinetic phase (which is the phase in which the speed of $60 \% \mathrm{~s}$ was achieved). The torque versus time curve was used to calculate TEMP $_{\text {torque }}$, defined as the time in seconds needed to reach the highest torque value. Because no significant differences were observed in the anthropometric characteristics of the study participants, no data normalization procedure was applied ${ }^{30}$.

\section{Data analysis}

Sample size calculation was performed using the software $\mathrm{G}^{*}$ Power (version 3.1.2; University of Trier, Trier, Germany), considering the type one error ( $\alpha=0.05$ ) and the type two error $(\beta=0.20)$ to detect the moderate effect $\left(\mathrm{f}^{2}>0.5\right)$. The calculations indicated that a sample of 33 subjects would be necessary.

Data are presented as the mean \pm standard deviation, and the data normality was verified using the Kolmogorov-Smirnov method. The independent variables were groups (REC, TRA and CON) and time (pre- and post-training). The dependent variables were RAD, PT and TEMP torque $_{\text {. An analysis }}$ of variance (ANOVA) mixed model 3 x 2 (protocol $\mathrm{x}$ time) was used with Tukey's post-hoc test. The Mauchly's sphericity test was applied, and when refuted, the analysis was based on the GreenhouseGeisser correction. The adopted significance was $5 \%$ $(\mathrm{p}<0.05)$, and the program used was SPSS (Statistical Package for Social Sciences), version 17.0.

\section{Results}

A total of 33 eligible subjects participated in this study. However, after randomization, three subjects who had been allocated to the CON group missed the after-test session, and a subject from the REC group missed the last training session. All of the remaining 29 subjects completed the exercise sections as originally assigned and were included in the subsequent analyses (Figure 2).

The pre- and post-training PT values (three sessions) of RE are shown in Table 2. No significant differences $(p>0.05)$ were observed between the groups for the pre-test. However, there was a significant gain in the PT post-training for the REC and TRA groups at $60 \%$ s and for the REC group at $180 \%$ s $(\mathrm{p}<0.001)$. At both of the velocities evaluated, a comparison between the groups revealed a higher gain in PT in the REC group. After training, the REC group showed increases of $6.14 \%$ at $60 \%$ and $9.56 \%$ at $180 \%$, which were significantly superior $(\mathrm{p}<0.05)$ to the gains of the TRA group $(4.47 \%$ at $60 \%$ s and $0.58 \%$ at $180^{\circ} / \mathrm{s}$ ).

The pre- and post-test RAD values are shown in Table 3. There was a significant difference in RAD for the REC and TRA groups ( $p=0.02$ ) after three sessions of RE, with a reduction in RAD. Conversely, the CON group showed an increase in RAD during the post-test analysis. In the intergroup analysis, a reduction in RAD was observed compared to $\mathrm{CON}$, which was similar between the training groups ( $>00.05)$.

The TEMP ${ }_{\text {torque }}$ values are shown in Table 3. It was observed that only the REC group reached PT significantly more rapidly ( $\mathrm{p}=0.008)$, with a decrease in TEMP ${ }_{\text {torque }}$ of $21.8 \%$ after three sessions of RE. Intergroup analysis revealed that this decrease in TEMP $_{\text {torque }}$ after the test was significant compared to that of the CON group ( $\mathrm{p}=0.009$ ), but the TRA and $\mathrm{CON}$ groups did not show a difference $(\mathrm{p}>0.05)$.

\section{Discussion}

The goal of the present study was to evaluate the effects on the neuromuscular responses of healthy young men of a short-term RE program (three sessions) that used reciprocal exercises compared to 


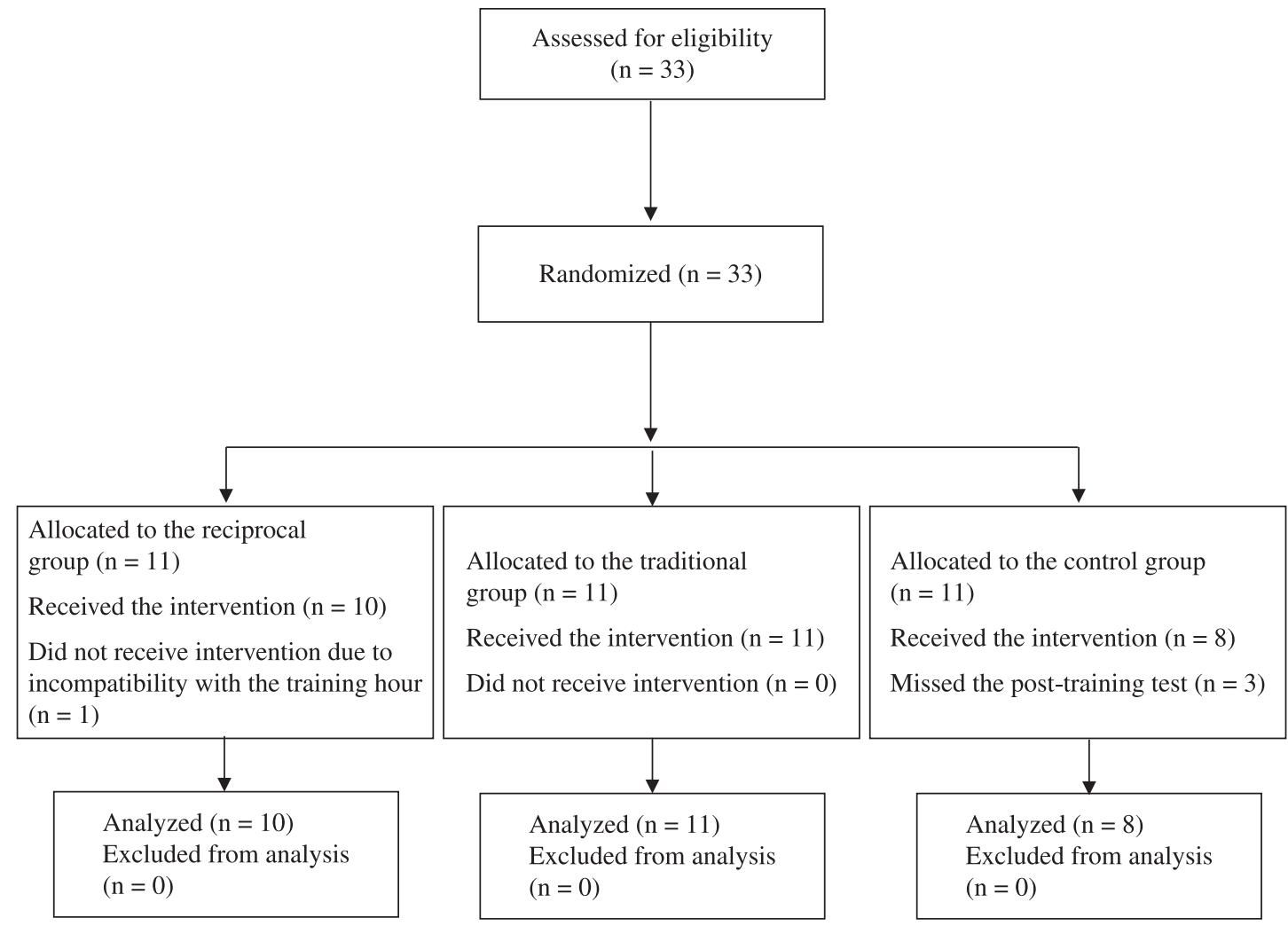

Figure 2. Flow diagram of study.

Table 2. The reciprocal (RA), traditional (TRA) and control (CON) groups peak torque (PT) during isokinetic contractions of knee extensors performed prior to and after short-term resistance training at $60 \% \mathrm{~s}$ and $180 \%$ s. Data are shown as the mean \pm standard deviation.

\begin{tabular}{lcccccc}
\hline & \multicolumn{2}{c}{ PT $(\mathbf{N} \cdot \mathbf{m}) / \mathbf{6 0} \% \mathbf{s}$} & & \multicolumn{3}{c}{$\mathbf{P T}(\mathbf{N} \cdot \mathbf{m}) / \mathbf{1 8 0} / \mathbf{s}$} \\
& Pre-training & Post-training & $\Delta \%$ & Pre-training & Post-training & $\Delta \%$ \\
RA & $239.15 \pm 26.34$ & $253.86 \pm 43.05$ & $6.14 *+$ & $162.64 \pm 25.15$ & $178.19 \pm 26.03$ & $9.56^{*} \ddagger$ \\
TRA & $206.71 \pm 14.76$ & $215.94 \pm 14.92$ & $4.47^{*}$ & $153.50 \pm 15.20$ & $154.40 \pm 18.00$ & 0.58 \\
CON & $212.20 \pm 30.09$ & $209.36 \pm 26.66$ & -1.33 & $167.80 \pm 16.10$ & $165.10 \pm 18.90$ & -1.60 \\
\hline
\end{tabular}

$*$ Greater than pre-training $(p<0.05)$; $\neq$ Greater than CON and TRA $(p<0.05) . \Delta \%$ : variation between pre-training and post-training $\left[\left(\frac{\text { post }- \text { pre }}{\text { pre }}\right) \times 100\right]$

a traditional short-term RE program. The findings of the present study partially support the proposed hypothesis, as the REC group showed the highest strength gains, especially at the highest speed $\left(180^{\circ} / \mathrm{s}\right)$. Both the TRA and REC groups were able to improve muscle strength and performance (RAD and TEMP $\left.{ }_{\text {torque }}\right)$ at the lowest speed $\left(60^{\circ} / \mathrm{s}\right)$.

In this study, three RE sessions produced significant gains with respect to extensor strength, thereby corroborating previous studies ${ }^{8,10,11,16}$. The REC and TRA groups showed significant PT gain (6\% and $4 \%$, respectively) at $60 \%$ s. The gains of REC also showed transference to a higher speed of $180 \%$, characterized by a gain of approximately $9 \%$ in extension strength.

Coburn et al. ${ }^{11}$ adopted three RE sessions using a protocol identical to TRA and with the same volume (four series of ten repetitions); however, 
Table 3. The reciprocal (RA), traditional (TRA) and control (CON) groups rate of development acceleration (RDA) and time to peak torque $\left(\mathrm{TIME}_{\text {torque }}\right.$ ) of the knee extensors performed prior to and after short-term resistance training at $60^{\circ} / \mathrm{s}$. Data are shown as the mean \pm standard deviation.

\begin{tabular}{lcccccc}
\hline & & RDA (milliseconds) & & \multicolumn{3}{c}{ TIME $_{\text {torque }}$ (milliseconds) } \\
& Pre-training & Post-training & $\Delta \%$ & Pre-training & Post-training & $\Delta \%$ \\
RA & $80 \pm 20$ & $70 \pm 10$ & $-13.0^{*}+$ & $480 \pm 62$ & $375 \pm 77$ & $-21.8^{*} \neq$ \\
TRA & $80 \pm 10$ & $70 \pm 10$ & $-13.0^{*}+$ & $493 \pm 86$ & $444 \pm 77$ & -9.9 \\
CON & $80 \pm 10$ & $90 \pm 10$ & $13.0^{*}$ & $497 \pm 45$ & $511 \pm 32$ & 3.0 \\
\hline
\end{tabular}

*Lower than pre-training $(p<0.05)$; $\neq$ Lower than $\operatorname{CON}(p<0.05) . \Delta \%$ : variation between pre-training and post-training - $\left[\left(\frac{\text { post }- \text { pre }}{\text { pre }}\right) \times 100\right]$.

the velocities were different $(30 \%$ s and $270 \%$ s). The authors reported that the group trained at $30 \%$ obtained strength gains both at the training speed and at $270 \%$ s (24\% and $11 \%$, respectively), which contradicts our findings for the TRA group, that they experienced no gains at the highest velocity. In the Coburn et al. ${ }^{11}$ study, a $40 \%$ increase in strength was observed only in the group that trained at $270 \%$ s. There is a discrepancy between the PT reported by Coburn et al. ${ }^{11}$ and the findings of the present study. This discrepancy may be due to the velocity adopted in the training portion of the Coburn study $\left(30^{\circ} / \mathrm{s}\right.$ and $2700 \%$ s) relative to that of the present study $(60 \%)$. In addition, the authors used a two-minute interval between sets, which could have allowed a recovery of extensor muscles of the knee and favored higher rates of $\mathrm{PT}^{31}$, compared to our study, in which the participants took only one-minute rest intervals.

Prevost et al. ${ }^{8}$ observed effects on muscle strength from two sessions of isokinetic RE in individuals trained at $30 \%$ s and $270 \%$ s, in a knee extension protocol that included three sets of ten repetitions. However, a gain of $22 \%$ in PT was observed only for the group that trained at the higher velocity. The magnitude of the strength gains achieved in Prevost et al. ${ }^{8}$ and Coburn et al. ${ }^{11}$ differ from the findings of the present study because controlling the acceleration phase of the motion may have been overlooked by the authors ${ }^{10}$. According to Brown and Whitehurst ${ }^{10}$, failure to control the acceleration phase may be responsible for differences in strength. This parameter was monitored and found to be responsive to the short training period in the present study. Unlike in the present study, Prevost et al. ${ }^{8}$ and Coburn et al. ${ }^{11}$ did not carry out familiarization before starting the training. It should also be considered that
Prevost et al. ${ }^{8}$ did not report any rest intervals between sets, which further complicates the explanation of the differences in strength gains that were observed.

The findings regarding the time necessary to reach PT indicated that the subjects treated with reciprocal RE were able to achieve PT $22 \%$ faster after three sessions. According to Widrick et al..$^{32}$, adaptations to $\mathrm{RE}$ are complex because they involve neural and peripheral mechanisms, and muscle function can be optimized by increasing the contractile ability of the muscle fibers (TEMP ${ }_{\text {torque }}$ ). Therefore, pre-stretching the knee extensor muscles by bending prior to each repetition in the reciprocal action modality may be responsible for producing the greatest gains in strength and the lowest TEMP ${ }_{\text {torque }}{ }^{33}$. One of these mechanisms is the storage and reuse of elastic energy and potentiation of the muscle contractile elements. The findings at $180 \%$ s reinforce this interpretation, considering that only the REC group showed a significant PT gain after the RE program.

Regarding RAD, significant gains were observed in both the REC and TRA groups. Brown and Whitehurst ${ }^{10}$ showed that after two RE training sessions at $60 \%$ and $240 \%$, (each with three sets of eight repetitions), an increase was generated in the rate of acceleration of the inferior limbs. Although Brown and Whitehurst ${ }^{10}$ did not observe an increase in strength, RAD and TEMP ${ }_{\text {torque }}$ are important variables. However, compared to TEMP ${ }_{\text {torque }}$ RAD represents only the ability to generate velocity and acceleration and may indicate qualitative changes, such as an increase in motor neuron recruitment or an increase in firing frequency. Cormie et al. ${ }^{35}$ showed that the musculoskeletal force-velocity relationship represents an important property to determine the capacity for potential energy production during a 
muscle contraction. One of the factors that determines this ability is the muscle fiber shortening velocity, which in turn may be enhanced by increased neural activation. The findings of this study therefore suggest that the neural adaptations resulting from three RE sessions generated effects in RAD, but these effects were independent of the order of exercise of the agonistic and antagonist muscles.

A limitation of our study was the exclusive use of $60 \%$ as the velocity in the analysis of RDA and $\mathrm{TEMP}_{\text {torque }}$. It is possible that an analysis during high velocity contractions would have yielded additional information on neuromuscular performance. Another limitation was the fact that the analysis was focused exclusively on the repetitions with higher PT. Perhaps the analysis of other variables, such as the total work and strength behavior throughout the RE session, would have provided a better representation of human performance.

The training at $60 \%$ demonstrated that gain can be transferred to higher velocities $(108 \%$ ), as shown with the REC group. This finding may be important because it implies that strength gains associated with higher velocities are related to an increase in the functional capacity in humans ${ }^{36}$. Therefore, one can expect that reciprocal RE can generate functional benefits, such as improvements to agility, balance and the activities of daily living that require use of the lower limbs. Furthermore, the results indicate that gains in muscular performance can be achieved even after short periods of training and that reciprocal contraction can bring additional benefits to neuromuscular performance. Future studies are needed to verify the effects of this type of $\mathrm{RE}$ on the performance of functional tests that are used in clinical practice. Furthermore, a study of the benefits of training with eccentric actions on a reciprocal mode would be interesting (considering the importance of eccentric action for the knee flexors and extensors) while performing daily activities, such as sitting and descending stairs.

\section{- Conclusions}

Both training methods were effective at improving muscle strength and performance at the training velocity examined $\left(60^{\circ} / \mathrm{s}\right)$. The reciprocal action protocol produced the greatest strength gain at the highest velocity of muscle action $(180 \%$ s). These findings suggest that reciprocal action is an advantageous protocol for both rehabilitation and sports. Further studies using muscle activation and co-activation are important to better understand the benefits of reciprocal action exercises in improving neuromuscular responses.

\section{Acknowledgements}

We would like to thank the Foundation for Research Support of the Federal District (Fundação de Apoio à Pesquisa do Distrito Federal-FAPDF, process $n^{\circ}$. 2009/00212-2) for financial support.

\section{References}

1. Rutherford OM, Jones DA. The role of learning and coordination in strength training. Eur J Appl Physiol Occup Physiol. 1986;55(1):100-105. http://dx.doi.org/10.1007/ BF00422902

2. Delecluse C, Van Coppenolle H, Willems E, Van Leemputte M, Diels R, Goris M. Influence of high-resistance and high-velocity training on sprint performance. Med Sci Sports Exerc. 1995 Aug;27(8):1203-1209. PMid:7476066. http://dx.doi.org/10.1249/00005768-199508000-00015

3. Bottaro M, Machado S, Nogueira W, Scales R, Veloso J. Effect of high versus low-velocity resistance training on muscular fitness and functional performance in older men. Eur J App Physiol. 2007;99(3):257-264. PMid:17146693. http://dx.doi.org/10.1007/s00421-006-0343-1

4. American College of Sports Medicine-ACSM. American College of Sports Medicine position stand. Progression models in resistance training for healthy adults. Med Sci Sports Exerc. 2009 Mar;41(3):687-708. PMid:19204579. http://dx.doi.org/10.1249/MSS.0b013e3181915670

5. American Association of Cardiovascular \& Pulmonary Rehabilitation - AACPR. Guidelines for Rehabilitation and secondary prevention programs. 3rd ed. Champaign: Human Kinetics; 1999.

6. Moritani T, De Vries HA. Neural factors versus hypertrophy in the time course of muscle strength gain. Am J Phys Med. 1979 June;58(3):115-130. PMid:453338.

7. Landin D, Nelson AG. Early phase strength development: a four-week training comparison of different programs. J Strength Cond Res. 2007 Nov;21(4):1113-1116. PMid:18076224.

8. Prevost MC, Nelson AG, Maraj BKV. The effect of two days of velocity-specific isokinetic training on torque production. J Strength Cond Res. 1999 Feb;13(1):35-39.

9. Akima H, Takahashi H, Kuno SY,Masuda K, Masuda T, Shimojo H, et al. Early phase adaptations of muscle use and strength to isokinetic training. Med Sci Sports Exerc. 1999 Apr;31(4):588-594. PMid:10211857. http:// dx.doi.org/10.1097/00005768-199904000-00016

10. Brown LE, Whitehurst M. The effect of short-term isokinetic training on force and rate of velocity 
development. J Strength Cond Res. 2003 Feb;17(1):88-94. PMid:12580662.

11. Coburn JW, Housh TJ, Malek MH, Weir JP, Cramer JT, Beck TW, et al. Neuromuscular responses to three days of velocity-specific isokinetic training. J Strength Cond Res. 2006 Nov;20(4):892-898. PMid:17194247.

12. Remaud A, Cornu C, Guevel A. Agonist muscle activity and antagonist muscle co-activity levels during standardized isotonic and isokinetic knee extensions. J Electromyogr Kinesiol. 2009 June;19(3):449-458. PMid:18093843. http://dx.doi.org/10.1016/j.jelekin.2007.11.001

13. Osternig LR. Isokinetic dynamometry: Implications for muscle testing and rehabilitation. Exerc Sport Sci Rev. 1986;14:45-80. PMid:3525192.

14. Molinari F, Knaflitz M, Bonato P, Actis MV. Electrical manifestations of muscle fatigue during concentric and eccentric isokinetic knee flexion-extension movements. IEEE Trans Biomed Eng. 2006;53(7):13091316. PMid:16830935. http://dx.doi.org/10.1109/ TBME.2006.873680

15. Avila MA, Brasileiro JS, Salvini TF. Electrical stimulation and isokinetic training: effects on strength and neuromuscular properties of healthy young adults. Rev Bras Fisioter. 2008;12(6):435-440. http://dx.doi. org/10.1590/S1413-35552008005000006

16. Beck TW, Housh TJ, Johnson GO,Weir JP, Cramer JT, Coburn JW, et al. Effects of two days of isokinetic training on strength and electromyographic amplitude in the agonist and antagonist muscles. J Strength Cond Res. 2007 Aug;21(3):757-762. PMid:17685705.

17. Bohannon RW. Knee extension torque during repeated knee extension-flexion reversals and separated knee extensionflexion dyads. Phys Ther. 1985 July;65(7):1052-1054. PMid:4011683.

18. Miller JP, Croce RV, Hutchins R. Reciprocal coactivation patterns of the medial and lateral quadriceps and hamstrings during slow, medium and high speed isokinetic movements. J Electromyogr Kinesiol. 2000 Aug;10(4):233-239. http:// dx.doi.org/10.1016/S1050-6411(00)00012-2

19. Burke DG, Pelham TW, Holt LE. The influence of varied resistance and speed of concentric antagonistic contractions on subsequent concentric agonistic efforts. J Strength Cond Res. 1999;13(3):193-197.

20. Jeon HS, Trimble MH, Brunt D, Robinson ME. Facilitation of quadriceps activation following a concentrically controlled knee flexion movement: the influence of transition rate. J Orthop Sports Phys Ther. 2001 Mar;31(3):122-129. PMid:11297017.

21. Carregaro RL, Cunha RR, Cardoso JR, Pinto RS, Bottaro $M$. Effects of different methods of antagonist muscles preactivation on knee extensors neuromuscular responses. Rev Bras Fisioter. 2011 Dec;15(6):452-459. http://dx.doi. org/10.1590/S1413-35552011005000028

22. Baker D, Newton RU. Acute effect on power output of alternating an agonist and antagonist muscle exercise during complex training. J Strength Cond Res. 2005 Feb;19(1):202-205. PMid:15705035.
23. Roy MA, Sylvestre M, Katch FI, Katch VL, Lagasse PP. Proprioceptive facilitation of muscle tension during unilateral and bilateral knee extension. Int J Sports Med. 1990 Aug;11(4):289-292. PMid:2228358. http:// dx.doi.org/10.1055/s-2007-1024809

24. Robbins DW, Young WB, Behm DG, Payne WR. The effect of a complex agonist and antagonist resistance training protocol on volume load, power output, electromyographic responses, and efficiency. J Strength Cond Res. 2010 July;24(7):1782-1789. PMid:20555283. http://dx.doi.org/10.1519/JSC.0b013e3181dc3a53

25. Blazevich AJ, Gill ND, Deans N, Zhou S. Lack of human muscle architectural adaptation after short-term strength training. Muscle Nerve. 2007 Jan;35(1):78-86. PMid:17039464. http://dx.doi.org/10.1002/mus.20666

26. Davies GJ, Heiderscheit B, Brinks K. Test interpretation. In: Brown LE. Isokinetics in human performance. 1st ed. Champaign: Human Kinetics; 2003.

27. Stumbo TA, Merriam S, Nies K, Smith A, Spurgeon D, Weir JP. The effect of hand-grip stabilization on isokinetic torque at the knee. J Strength Cond Res. 2001 Aug;15(3):372-377. PMid:11710668.

28. Cunha R, Martorelli AS, Carregaro RL, Bottaro M. Treinamento isocinético de curto prazo promove aumento da força muscular em indivíduos jovens. Motriz. 2011;17(1):138-144.

29. Celes R, Brown LE, Pereira MC, Schwartz FP, Rocha Junior VA, Bottaro M. Gender muscle recovery during isokinetic exercise. Int J Sports Med. 2010 Dec;31(12):866-86. PMid:21072739. http://dx.doi. org/10.1055/s-0030-1254156

30. Ugrinowitsch C, Tricoli V, Rodacki AL, Batista M, Ricard MD. Influence of training background on jumping height. J Strength Cond Res. 2007 Aug;21(3):848-852. PMid:17685694.

31. Pincivero DM, Campy RM. The effects of rest interval length and training on quadriceps femoris muscle. Part I: knee extensor torque and muscle fatigue. J Sports Med Phys Fitness. 2004 June;44(2):111-118. PMid:15470307.

32. Widrick JJ, Stelzer JE, Shoepe TC, Garner DP. Functional properties of human muscle fibers after short-term resistance exercise training. Am J Physiol Regul Integr Comp Physiol. 2002 Aug;283(2):R408-416. PMid:12121854.

33. Markovic G, Mikulic P. Neuro-musculoskeletal and performance adaptations to lowerextremity plyometric training. Sports Med. 2010 Oct;40(10):859-895. PMid:20836583. http://dx.doi. org/10.2165/11318370-000000000-00000

34. Aagaard P, Simonsen EB, Andersen JL, Magnusson P, Dyhre-Poulsen P. Incresed rate of force development and neural drive of human skeletal muscle following resistance training. J Appl Physiol. 2002 Oct;93(4):1318-1326. PMid:12235031.

35. Cormie P, McGuigan MR, Newton RU. Developing maximal neuromuscular power: Part 1--biological basis of maximal power production. Sports 
Med. 2011;41(1):17-38. PMid:21142282. http://dx.doi. org/10.2165/11537690-000000000-00000

36. Tschopp M, Sattelmayer MK, Hilfiker R. Is power training or conventional resistance training better for function in elderly persons? A meta-analysis. Age Ageing. 2011 Sept;40(5):549-556. PMid:21383023. http://dx.doi. org/10.1093/ageing/afr005

\section{Correspondence}

\section{Rodrigo L. Carregaro}

Universidade de Brasília (UnB)

Campus UnB Ceilândia, QNN 14 Área Especial - Ceilândia Sul CEP 72220-140, Brasília, DF, Brasil

e-mail: rodrigocarregaro@unb.br 